\title{
Centroamérica frente a la pandemia: retos de la política macroeconómica ${ }^{1}$
}

\author{
Juan Carlos Moreno Brid y Rodrigo Alfonso Morales López
}

\section{Resumen}

El propósito del presente artículo es doble. Por una parte, se ocupa de describir el estado socioeconómico de la subregión centroamericana antes de la pandemia de enfermedad por coronavirus (COVID-19). Por otra, pone a disposición un análisis comparativo de las políticas macroeconómicas que han aplicado los gobiernos de los países ante la crisis derivada de la pandemia. Como un aspecto central el texto también plantea, desde una perspectiva estructural, los principales desafíos en términos de políticas nacionales (durante la pandemia y posteriores a ella, si cabe así decirlo) de protección social y de reactivación de la actividad productiva y el empleo, así como la necesidad de lograr un pacto nacional por una nueva agenda de desarrollo. Se ponen de relieve aspectos externos que deberían acompañar a las políticas nacionales, entre ellas las referidas a coordinación regional, cooperación y financiamiento internacional, así como una reestructuración de las instituciones financieras y crediticias.

\section{Palabras clave}

COVID-19, virus, epidemias, aspectos económicos, macroeconomía, política fiscal, política monetaria, producto interno bruto, exportaciones, turismo, remesas, pobreza, cooperación regional, desarrollo económico, Centroamérica

\section{Clasificación JEL}

E52, E60, E62, H60

\section{Autores}

Juan Carlos Moreno Brid es Profesor Titular de la Facultad de Economía de la Universidad Nacional Autónoma de México (UNAM). Correo electrónico: juancarlosmorenobrid@gmail.com.

Rodrigo Alfonso Morales López es Investigador Posdoctoral de la Facultad de Economía de la Universidad Nacional Autónoma de México (UNAM). Correo electrónico: ramorales88@gmail.com.

\footnotetext{
1 Los autores agradecen la valiosa colaboración brindada por Abraham Pazos Rodríguez como asistente de investigación.
} 


\section{Introducción}

La pandemia de COVID-19 ha ocasionado una doble tragedia mundial en términos de, por una parte, muertes y enfermedad y, por otra, colapso de la actividad productiva y el empleo y deterioro de las condiciones de vida de los pueblos. Su impacto sume a las economías de América Latina en la depresión más aguda en casi 100 años. Para Centroamérica el impacto es devastador. El confinamiento temporal - y no se sabe si será recurrente - la golpeó brutalmente, dada la elevada incidencia de la pobreza, una lacerante desigualdad y una alta prevalencia del trabajo informal mal remunerado, sin adecuada seguridad médica ni ahorros de reserva para compensar la pérdida de ingresos. Además, por tratarse de economías pequeñas, muy abiertas y escasamente industrializadas, su desempeño depende en forma crucial del sector externo, hoy detenido. A esta restricción de la balanza de pagos se debe agregar la presión sobre la conducción de la política macroeconómica impuesta por la volatilidad de los capitales y las condiciones que establecen en forma continua las agencias calificadoras de riesgo, que modifican de un día a otro las posibilidades de acceso al financiamiento internacional.

Los Gobiernos del istmo percibieron pronto la gravedad de la pandemia y sus repercusiones. Con excepción de Nicaragua, declararon el estado de emergencia o un estado similar de alarma nacional. Tomando lecciones de la teoría y la práctica de la macroeconomía ante pandemias, en primer lugar, adoptaron medidas de salvaguarda para fortalecer el sector de la salud, brindaron información sanitaria y ordenaron medidas de confinamiento y distanciamiento físico, entre otras. En segundo lugar, pusieron en marcha una política fiscal y monetaria contracíclica.

El propósito de este artículo es examinar el estado de la política macroeconómica en los países de Centroamérica frente a la pandemia. En el segundo apartado, se señalan los rasgos principales de la situación económica de Centroamérica justo antes de la irrupción de la emergencia sanitaria y la subsecuente crisis internacional y se identifican los canales de transmisión por los cuales la crisis del COVID-19 impacta a la subregión. En el tercer acápite, se examinan las principales respuestas en materia de política macroeconómica que, en diversos grados y alcances, están dando los Gobiernos de Centroamérica. El trabajo finaliza con una serie de reflexiones sobre las lecciones que dejan a la subregión la pandemia y la crisis económica derivada de ella. Sin adelantar conclusiones, puede afirmarse que estamos ante una dramática llamada de atención que obliga a modificar la pauta de desarrollo que han seguido tanto la subregión como el mundo. Reaccionar ante este aviso obliga a convocar con carácter de urgencia a un pacto regional por un crecimiento persistente, sustentable e incluyente, por una nueva agenda de desarrollo.

\section{Centroamérica: antecedentes de la doble crisis y mecanismos de transmisión}

La pandemia encontró la economía de América Latina, a inicios de 2020, estancada. En promedio el producto interno bruto (PIB) real mantuvo en 2019 el mismo nivel que 12 meses atrás. Por su parte, la economía de Centroamérica registraba un moderado dinamismo. Su PIB real se expandió un 2,5\% en promedio, con diferencias importantes de un país a otro. Las economías de Guatemala y Panamá crecieron un poco más del 3,0\%. Las de Costa Rica, El Salvador y Honduras se expandieron un poco menos, entre un 2,0\% y un 3,0\%. Un gran contraste lo presenta Nicaragua, cuya economía se contrajo un 3,9\%, entrampada en la profunda crisis económica y política que se inició en 2018 y redundó en una caída del PIB del 4\% ese año (CEPAL, 2020a).

Un elemento importante detrás del desempeño de la subregión es la balanza de pagos. Por tratarse de economías pequeñas muy abiertas, su desempeño depende de su forma de inserción en 
los mercados globalizados. Por lo tanto, en la medida en que el comercio mundial no cobra impulso, la subregión no logra un dinamismo económico. Un segundo elemento detrás del lento crecimiento es la falta de impulso sostenida de la formación bruta de capital fijo. Según datos de CEPALSTAT (CEPAL, 2020i), la inversión real se contrajo en promedio un 1,7\% en 2019, afectada por la incertidumbre generada por las tensiones comerciales entre los Estados Unidos y China, la evolución del brexit, la volatilidad del precio del petróleo y el mayor proteccionismo en el mundo desarrollado. El panorama se complicó aún más con la crisis política de Nicaragua, ciertos problemas de estabilidad institucional en Honduras y tensiones políticas en Costa Rica.

En cuanto a las finanzas públicas, en 2019, de acuerdo con datos de la Comisión Económica para América Latina y el Caribe (CEPAL, 2020b), en América Latina el déficit público fue en promedio del 3,1\% del PIB. En comparación con ese promedio, solo Costa Rica y Panamá presentaron niveles superiores (6,9\% y 3,7\% respectivamente). El espacio fiscal, en cierta medida reflejado por el cociente entre la deuda pública y el PIB, era heterogéneo en la subregión, con un promedio del 47,5\%. En Guatemala era del 26,1\%, en Nicaragua del 37,5\%, en Panamá del 46,4\% y en Honduras del 49,1\%. Por su parte, El Salvador $(67,4 \%)$ y Costa Rica $(58,5 \%)$ estaban relativamente más restringidos en este terreno, lo que condiciona, si bien no determina, las posibilidades de aplicar una política contracíclica. La inflación, por su parte, estaba bajo control desde hacía tiempo.

En el terreno social, según datos de CEPALSTAT (CEPAL, 2020i) y CEPAL (2020a), en la subregión la pobreza mostraba una tendencia a la baja entre 2012 y 2019, con excepción de Nicaragua. Empero, su incidencia seguía siendo alta: en El Salvador llegaba al 33,7\%, en Guatemala al 48,6\%, en Honduras al $54,8 \%$ y en Nicaragua al $47,1 \%$. Solo Costa Rica (16,5\%) y Panamá $(14,6 \%)$ registraban porcentajes inferiores al 20\%. La desigualdad es similar en los países de la subregión. En El Salvador, el coeficiente de Gini de la distribución del ingreso era 0,40 en 2018, en Honduras 0,48, en Costa Rica 0,49 y en Panamá 0,50. Por su parte, Guatemala mostraba un valor de 0,54 y Nicaragua uno de 0,50 en 2014, último dato disponible en el caso de estos países.

En resumen, al enfrentar la crisis la subregión acusaba un crecimiento moderado, sin presión inflacionaria alguna. La situación de las finanzas públicas, aunque compleja y con un espacio fiscal acotado, no presentaba tensiones preocupantes. Se registraban altos niveles de pobreza, desigualdad y malnutrición, y se contaba con un sistema de salud poco robusto. El reto que impuso la pandemia supera ampliamente el de la crisis financiera mundial del período 2008-2009 (Banco Mundial, 2020).

La pandemia iniciada en China tuvo sus primeros impactos en sus países vecinos y en el mundo industrializado. Estos cobraron mayor gravedad en economías en desarrollo como las centroamericanas. Por una parte, sus alarmantes niveles de pobreza y desigualdad se agudizaron abruptamente por el confinamiento y la caída de los empleos y las remuneraciones. En muchas economías se revertirán los progresos de años en el combate a la pobreza y en materia de cobertura educativa y otras áreas. Entre las razones del mayor impacto en algunos países están las insuficiencias crónicas de los sistemas de seguridad social, las condiciones de morbilidad de sus poblaciones, la falta de estabilizadores automáticos, la vasta informalidad laboral y la amplitud del sector informal, precario y escasamente bancarizado.

Además, la subregión adolece de una carga tributaria baja y regresiva, que en varios países tiende a depender de ingresos derivados de la exportación de bienes primarios, volátiles en sus cotizaciones en los mercados internacionales. Su pauta de inserción en la economía global, cimentada en la exportación de productos básicos o en la maquila, acentúa el papel de la balanza de pagos como restricción al crecimiento de las economías centroamericanas. Tal dependencia de la demanda externa y los términos de intercambio se traduce en incapacidad para alcanzar un crecimiento económico sostenido a las elevadas tasas que se requieren para absorber la creciente mano de obra y reducir la precariedad laboral. 
La pandemia provocó un choque de extraordinaria magnitud, golpeando la oferta y la demanda agregadas. Afecta, también, los flujos de fondos interinstitucionales y las hojas de balance de empresas y familias, el clima de negocios y la inversión local y extranjera, y empodera aún más a las agencias calificadoras de riesgo. Un primer impacto en la subregión se produce con las medidas de confinamiento, distanciamiento físico y restricción a la movilidad. La suspensión de labores, el cierre de empresas y los recortes de ingresos colapsan el gasto y deprimen más la actividad productiva. Minan el bienestar de toda la población - aunque no en la misma magnitud -, incluidas las clases medias, al perderse trabajos formales y prestaciones. Su resultado es un mayor nivel de pobreza y desigualdad.

La restricción externa al crecimiento económico de la subregión cobra fuerza en la pandemia. Su impacto se transmite a través de los diversos componentes de la balanza de pagos, tanto en la cuenta corriente como en la de capital. En particular, el confinamiento y la recesión en el mundo desarrollado - los Estados Unidos, en especial - detienen las exportaciones, interrumpen cadenas globales de suministros, colapsan el turismo y las remesas. En síntesis, debilitan las principales fuentes de divisas de Centroamérica. La situación se complica con la caída de los flujos de inversión extranjera, en particular hacia economías en desarrollo. A ello se suman fugas de capitales y un difícil acceso al financiamiento internacional. Si bien las importaciones se reducen debido a la disminución de la actividad, la balanza de pagos tensa las posibilidades de reactivación económica. Ello pone en especial riesgo a las economías dolarizadas de El Salvador y Panamá, impedidas de modificar el tipo de cambio nominal para amortiguar choques externos.

Cabe ilustrar algunos aspectos de la vulnerabilidad señalada de Centroamérica a los impactos de la pandemia. Sus exportaciones como proporción del PIB son mayores, en promedio, que las del resto de América Latina (véase el cuadro 1). El rango de la contribución de las exportaciones oscila entre el 22,2\% del PIB (Guatemala) y el 51,2\% del PIB (Panamá). De acuerdo con el Sistema de Estadísticas de Comercio (SEC) de la Secretaría de Integración Económica Centroamericana (SIECA, 2020), el 34\% del total exportado de bienes de la subregión fue a los Estados Unidos en 2019. Destaca la relevancia del comercio intrarregional. En efecto, las ventas entre los propios países de la subregión ascendieron al $31 \%$ de sus exportaciones totales de bienes $^{2}$.

Cuadro 1

Centroamérica: contribución de las exportaciones, el turismo y las remesas al PIB, 2019

(En porcentajes del PIB)

\begin{tabular}{lccr}
\hline País & Exportaciones & Turismo & \multicolumn{2}{c}{ Remesas } \\
\hline América Latina & 20,4 & 8,1 & $1,7^{\mathrm{a}}$ \\
\hline Costa Rica & 37,5 & 12,0 & 0,8 \\
\hline El Salvador & 29,7 & 11,0 & 20,9 \\
\hline Guatemala & 22,2 & 6,2 & 13,7 \\
\hline Honduras & 45,7 & 11,7 & 21,6 \\
\hline Nicaragua & 49,0 & 10,1 & 13,2 \\
\hline Panamá & 51,2 & 13,6 & 0,7 \\
\hline
\end{tabular}

Fuente: Elaboración propia, sobre la base de Comisión Económica para América Latina y el Caribe (CEPAL), CEPALSTAT, 2020 [base de datos en línea] http://interwp.cepal.org/cepalstat/Portada.html?idioma=spanish; CEPAL, Estudio económico de Centroamérica y la República Dominicana en 2019 y perspectivas para 2020 (LC/MEX/TS.2020/29), Ciudad de México, 2020, y Consejo Mundial de Viajes y Turismo, "Economic Impact Reports" [en línea] https://wttc.org/Research/ Economic-Impact.

a Dato correspondiente a 2018.

2 Las cifras proporcionadas por la SIECA no incluyen las exportaciones de las zonas francas y la maquila. 
El debilitamiento de la demanda externa también presiona, a la baja, los precios de una gran variedad de productos básicos y otros productos de la subregión. Sin embargo, en las cifras del período enero-mayo de 2020 se registra un incremento interanual de los precios de algunos productos clave de exportación. Por ejemplo, en Costa Rica se registró un incremento de los precios de las exportaciones de dispositivos médicos, mientras que Guatemala, Honduras y Nicaragua informaron sobre un fenómeno similar en los precios de algunos bienes agrícolas o minerales de exportación, por ejemplo, el banano o el oro.

La exportación de bienes agrícolas resulta hasta cierto punto menos perjudicada, debido a la menor elasticidad respecto del ingreso de la demanda externa de alimentos (CEPAL, 2020c). Es todavía muy pronto para saber si el alza de precios de ciertos productos de exportación persistirá. Por otra parte, la caída de los precios de los energéticos reduce considerablemente el costo de las importaciones de Centroamérica. Al vigilar las tendencias de las cotizaciones de los bienes agropecuarios, minerales y petróleo habrá que poner atención en la evolución de los términos de intercambio de la subregión.

En las exportaciones de servicios, el principal impacto se percibe en el turismo, ante las restricciones a la movilidad y el riesgo de contagio al viajar. No se ven perspectivas de una recuperación robusta en el corto plazo. En la reanudación paulatina de esta actividad se esperaría que se prefieran trayectos cortos, lo que podría impulsar el turismo interno y regional. Los países que exhiben la mayor dependencia del turismo, en cuanto a su contribución al PIB, son Panamá (13,6\%), Costa Rica (12,0\%) y Honduras (11,7\%). Igualmente importante es su aporte en el empleo y las exportaciones de servicios. En el período 2014-2018, el turismo representó en Panamá el 22,4\% de dichas exportaciones, y en ese mismo lapso participó con el 12,2\% del empleo en Costa Rica (Mooney y Zegarra, 2020).

La probable disminución de las remesas familiares, debido al deterioro del mercado laboral estadounidense, es otro golpe para Centroamérica, si bien alcanza distinta intensidad en los diferentes países. Por un lado, en El Salvador, Guatemala, Honduras y Nicaragua, las remesas, medidas como proporción del PIB, se ubicaron en 2019 en un rango entre el 13,2\% (Nicaragua) y el 21,6\% (Honduras). En Costa Rica y Panamá, no llegan al 1\% del PIB. Ambos países, en períodos de dinamismo económico, tienden a ser más origen que destino de transferencias familiares. Después de una caída pronunciada, las remesas dirigidas a El Salvador, Guatemala y Honduras han mostrado una recuperación desde junio de 2020 (CEPAL, 2020a). Su evolución en la segunda parte del año está sujeta a condicionantes difíciles de predecir. Está el riesgo que representa la situación sanitaria, económica e incluso política en los Estados Unidos y la incertidumbre sobre la continuidad de los apoyos especiales a los trabajadores, pilar importante de su política contracíclica. Finalmente, las variaciones cambiarias también incidirán en el poder de compra en países de destino.

A la vez, las interrupciones en las cadenas globales de valor a consecuencia de la pandemia están mermando el acceso de Centroamérica a insumos intermedios para la producción de bienes de consumo interno o exportaciones. El Salvador, Honduras y Nicaragua son los países de la subregión que más dependen de insumos importados. Panamá, por su parte, verá una reducción considerable de los ingresos derivados de las operaciones del Canal y la Zona Libre de Colón (Castellani y otros, 2020).

Más allá de los efectos mencionados, la pandemia ha provocado una mayor volatilidad y percepción de riesgo en los mercados financieros. El índice de bonos de mercados emergentes (EMBI) - la diferencia entre las tasas de interés que pagan los bonos emitidos por países en desarrollo y los bonos del Departamento del Tesoro de los Estados Unidos - muestra un deterioro en la subregión y en el mundo en desarrollo en general. Según datos del Banco Central de la República Dominicana (2020), entre febrero y septiembre de 2020 el EMBI (riesgo país) de América Latina se incrementó un 31,8\%. El deterioro ha sido mayor en Centroamérica: el aumento del riesgo país en Guatemala fue del 31,7\%, en Honduras del 52\%, en Panamá del 52,9\%, en Costa Rica del 54,7\% y en El Salvador del 111,7\%. El riesgo país de El Salvador (841 puntos básicos) es el más alto de la subregión - para Nicaragua no 
hay datos - , seguido por los de Costa Rica (667), Honduras (398), Guatemala (323) y Panamá (203), frente a un agregado de 441 puntos básicos para América Latina en su conjunto. Seguramente en el resto del año la dificultad para acceder al financiamiento externo será creciente y parece imposible que las bajas tasas de interés en los mercados internacionales compensen el mayor riesgo país en el costo del financiamiento a la subregión.

Lo anterior incide de manera negativa en la inversión extranjera directa (IED) a Centroamérica y, en un momento dado, puede ser un detonante de una fuga masiva de capitales. Ya era alarmante que en el primer trimestre de 2020 - cuando la pandemia todavía no se dejaba sentir con fuerza - las cifras oficiales registraran caídas de la IED en todos los países centroamericanos. Según datos de CEPALSTAT (CEPAL, 2020i), la caída interanual de la IED en ese período era del 90,9\% en El Salvador, el 11,6\% en Guatemala, el 9,1\% en Costa Rica, el 5,1\% en Panamá y el 1,5\% en Honduras. Es previsible que la caída se agudice en el resto del año.

La coyuntura repercute en el estado de las finanzas públicas. Por una parte, los gastos se elevan para atender a las necesidades que surgen ante la pandemia; por otra, los ingresos se contraen con el menor impulso económico interno y del comercio exterior. El endurecimiento de las condiciones de financiamiento complica las perspectivas. Asimismo, las depreciaciones cambiarias elevan el peso del cociente entre la deuda y el PIB, lo que tiende a restringir el espacio fiscal. En este contexto, los Gobiernos deberán procurar que la inversión pública no sea la variable de ajuste y que más bien se refuerce tanto para paliar la caída de la demanda en el presente como para avanzar en el mediano y largo plazo en una transformación de la estructura productiva a fin de insertarse en una senda de expansión elevada, sostenible e incluyente.

Los balances entre ahorro e inversión del sector privado también acusan los efectos de la crisis. Innegablemente el clima de inversión se hunde en la tormenta, afectado por la caída de las ventas, los cierres de actividades y la incertidumbre sobre la evolución de la pandemia y de variables clave de la economía nacional y mundial. El consumo privado, del mismo modo, está presionado a la baja en medio de la emergencia, como resultado de la caída de los ingresos y los empleos, y también por motivos de precaución.

Los efectos negativos se distribuyen de manera desigual en los sectores económicos y las poblaciones. La industria del turismo, la industria cultural, el comercio, los hoteles y restaurantes, el transporte y el sector textil se verán más afectados (CEPAL 2020e). Entre los grupos vulnerables destacan las mujeres, cuya situación resulta afectada por la falta de una economía del cuidado, a lo que se suma la violencia intrafamiliar, que tiende a exacerbarse en situaciones de confinamiento o contracción de ingresos. Los estratos de ingresos medios-bajos corren el riesgo de caer en situación de pobreza, lo que significaría borrar el avance material que la clase media tardó años en lograr. Los trabajadores informales -incluidos los que se desempeñan en el empleo doméstico- experimentan nuevas carencias y riesgos en materia de salud en la medida en que los programas de apoyo no los compensan. Los niños y los adolescentes ven que su acceso a educación de calidad se resquebraja o se destruye, sin mencionar el desarrollo integral que requieren en esas edades. Es posible que muchos abandonen sus estudios para buscar trabajo y compensar la pérdida de ingresos familiares. Se pueden seguir enumerando vulnerabilidades agudas en otros grupos: las personas mayores, la población rural, los indígenas y los afrodescendientes, las personas con discapacidad, los migrantes, las personas en situación de calle y otros (CEPAL, 2020f).

La magnitud final de los diferentes impactos de la crisis sobre las economías centroamericanas dependerá de la evolución de la pandemia, de las medidas que implementen los países y del diseño de políticas económicas oportunas para su posterior ejecución en el mediano y largo plazo. En la siguiente sección, se examinarán las respuestas de política económica, con énfasis en las políticas fiscal y monetaria, que los Gobiernos de la subregión han puesto en marcha para atender la emergencia. 


\section{La respuesta de política macroeconómica frente a la pandemia}

Los Gobiernos de la subregión, ante la gravedad de la situación provocada por la pandemia, comenzaron a tomar medidas inéditas. Movilizaron recursos para la salud, restringieron la movilidad, las actividades productivas y comerciales, y congelaron la vida comunitaria y social como la conocíamos. Siguiendo las lecciones de Keynes, implementaron políticas contracíclicas. En esta sección nos concentraremos en las políticas macroeconómicas más relevantes: la fiscal y la monetario-financiera ${ }^{3}$. El cuadro 2 ofrece una clasificación de ellas.

Cuadro 2

Centroamérica: políticas adoptadas ante la crisis provocada por el COVID-19

\begin{tabular}{|c|c|c|c|c|c|c|c|}
\hline Tipo de política & Medidas & Costa Rica & El Salvador & Guatemala & Honduras & Nicaragua & Panamá \\
\hline \multirow{6}{*}{ Política fiscal } & $\begin{array}{l}\text { Modificaciones temporales en la } \\
\text { recolección de impuestos }\end{array}$ & $x$ & $x$ & $x$ & $x$ & & $x$ \\
\hline & Transferencias públicas & $x$ & $x$ & $x$ & $x$ & $x$ & $x$ \\
\hline & Aplazamiento del pago de servicios & $x$ & $x$ & $x$ & $x$ & $x$ & $x$ \\
\hline & Aumento de salarios en el sector público & & $x$ & & & & \\
\hline & Inversión física & $x$ & $x$ & $x$ & $x$ & & $x$ \\
\hline & Emisión de deuda pública & $x$ & $x$ & $x$ & $x$ & & $x$ \\
\hline \multirow{7}{*}{$\begin{array}{l}\text { Política } \\
\text { monetaria y } \\
\text { crediticia }\end{array}$} & Reducción de la tasa de política monetaria & $x$ & & $x$ & $x$ & $x$ & \\
\hline & Modificación del encaje bancario & $x$ & $x$ & $x$ & & $x$ & \\
\hline & Otras medidas para proveer liquidez & $x$ & & $x$ & $x$ & & \\
\hline & Moratoria y reestructuración de préstamos & $x$ & $x$ & $x$ & $x$ & & $x$ \\
\hline & $\begin{array}{l}\text { Congelación de la calificación crediticia } \\
\text { y aceleración de procesos }\end{array}$ & $x$ & $x$ & & $\mathrm{x}$ & & \\
\hline & Creación de nuevas líneas de crédito & $x$ & $x$ & $x$ & $x$ & & $x$ \\
\hline & Intervención en el tipo de cambio & & & $x$ & & & \\
\hline \multirow{2}{*}{ Otras } & Control de precios & $x$ & $x$ & $x$ & $x$ & & $x$ \\
\hline & Intervención directa en el mercado laboral & $x$ & $x$ & $X$ & $x$ & & $x$ \\
\hline
\end{tabular}

Fuente: Elaboración propia, sobre la base de A. Matarrita y R. Romero, "Políticas económicas implementadas en la región CAPARD para contrarrestar el impacto del COVID-19", Notas Económicas Regionales, N 114, San José, Secretaría Ejecutiva del Consejo Monetario Centroamericano (SECMCA), junio de 2020; Comisión Económica para América Latina y el Caribe (CEPAL), "Observatorio COVID-19 en América Latina y el Caribe: impacto económico y social", 2020 [en línea] https://www.cepal.org/en/topics/covid-19; Fondo Monetario Internacional (FMI), "Policy Responses to COVID-19", 2020 [en línea] https://www.imf.org/en/Topics/imf-and-covid19/Policy-Responses-to-COVID-19; Banco Central de Honduras, "El Banco Central de Honduras revisa el programa monetario 2020-2021 y aprueba una nueva reducción en su tasa de política", Boletín de Prensa, № 42/2020, Tegucigalpa, 31 de julio de 2020; Banco de Guatemala, "La Junta Monetaria por unanimidad reduce en 25 puntos básicos la tasa de interés líder de política monetaria”, Boletín de Prensa, Ciudad de Guatemala, 25 de junio de 2020; Ministerio de Hacienda de El Salvador, Estimación de los costos económicos, sociales, fiscales y medidas adoptadas en el marco de la pandemia COVID-19, San Salvador, abril de 2020, y Ministerio de Trabajo y Desarrollo Laboral de Panamá, "Reglamentan la suspensión de los contratos de trabajo", 20 de marzo de 2020 [en línea] https://www.mitradel.gob.pa/reglamentan-la-suspension-de-los-contratos-de-trabajo-2/.

Costa Rica, Guatemala y Honduras hicieron uso de una amplia gama de políticas fiscales, monetarias y crediticias. En Nicaragua, la reacción ha sido muy débil e insuficiente. Panamá y El Salvador - dado que tienen economías dolarizadas - respondieron principalmente por el lado de los apoyos fiscales y crediticios. En el ámbito fiscal, como puede observarse en el cuadro 3, la orientación contracíclica en 2020 lleva a proyectar reducciones - con respecto a lo presupuestado - de los ingresos tributarios. Asimismo, se proyectan aumentos del gasto público, del déficit fiscal y de la deuda pública en relación

3 Un listado y una descripción en detalle de la amplia gama de respuestas de política económica y social que cada uno de los países de la región ha puesto en marcha ante la pandemia se encuentra en el Observatorio COVID-19 en América Latina y el Caribe, de la CEPAL [en línea] https://www.cepal.org/es/temas/covid-19. La información a nivel mundial se encuentra en los sitios web del Fondo Monetario Internacional (FMI) [en línea] https://www.imf.org/external/spanish/index.htm y el Banco Mundial [en línea] https://www.bancomundial.org/. 
con el PIB en todos los países de la subregión. En efecto, la combinación de la contracción de la actividad productiva y la instrumentación de políticas contracíclicas, tanto con aumentos del gasto como con reducciones - hasta cierto punto endógenas - de algunos ingresos tributarios, provocará un aumento considerable del cociente entre deuda pública y PIB en 2020. El aumento es mayor en las economías no dolarizadas en la medida en que se produce una depreciación cambiaria significativa.

Cuadro 3

Centroamérica: variación de los principales indicadores fiscales, 2020

(En porcentajes del PIB)

\begin{tabular}{lrrrrrr}
\hline Concepto & Costa Rica & El Salvador & Guatemala & Honduras & Nicaragua & Panamá \\
\hline Ingresos tributarios & & & & & & \\
\hline Presupuesto & 13,3 & 18,2 & 10,4 & 16,8 & 17,2 & 7,7 \\
\hline Estimación de cierre & 12,6 & 17,1 & 9,8 & 15,8 & 17,0 & 7,0 \\
\hline Pérdida esperada (puntos porcentuales) & 0,7 & 1,1 & 0,5 & 1,0 & 0,2 & 0,7 \\
\hline Gastos totales & & & & & & \\
\hline Presupuesto & 22,1 & 22,1 & 13,8 & 18,4 & 18,9 & 15,5 \\
\hline Estimación de cierre & 22,6 & 28,6 & 16,3 & 21,6 & 19,4 & 22,0 \\
\hline Aumento esperado (puntos porcentuales) & 0,5 & 6,5 & 2,5 & 3,2 & 0,5 & 6,4 \\
\hline Déficit fiscal & & & & & & \\
\hline$\quad$ Presupuesto & $-7,9$ & $-1,9$ & $-2,6$ & $-2,0$ & 0,0 & $-3,1$ \\
\hline Estimación de cierre & $-8,3$ & $-11,9$ & $-5,8$ & $-6,4$ & $-0,8$ & $-6,2$ \\
\hline Aumento esperado (puntos porcentuales) & $-0,4$ & $-10,0$ & $-3,3$ & $-4,4$ & $-0,9$ & $-3,1$ \\
\hline Deuda pública & & & & & & \\
\hline$\quad$ Presupuesto & 62,8 & 71,1 & 27,3 & 51,2 & 35,9 & 46,8 \\
\hline Estimación de cierre & 67,2 & 92,1 & 31,7 & 56,7 & 38,0 & 56,2 \\
\hline Aumento esperado (puntos porcentuales) & 4,5 & 21,0 & 4,4 & 5,6 & 2,0 & 9,3 \\
\hline
\end{tabular}

Fuente: Instituto Centroamericano de Estudios Fiscales (ICEFI), "Estimación de la situación fiscal de Centroamérica al final de 2020", Notas de Coyuntura, № 05-2020, Guatemala, julio de 2020.

Según proyecciones del Instituto Centroamericano de Estudios Fiscales (ICEFI, 2020b), la deuda de los gobiernos centrales se incrementará, en promedio para la subregión, 8 puntos porcentuales del PIB con respecto a los valores presupuestados, ubicándose en un 57\%. El mayor aumento, de 21 puntos porcentuales, corresponderá a El Salvador, que registraría un nivel de deuda por encima del 90\% del PIB. En los demás países, el aumento sería de un solo dígito; el mayor correspondería a Panamá (9 puntos porcentuales), seguido por Honduras (6 puntos porcentuales). Con ello, se proyecta que la deuda de ambos países llegue a un monto equivalente al $56 \%$ del PIB respectivo. Tanto en Guatemala como en Costa Rica se estima que dicho cociente presentará un alza de alrededor de 4,5 puntos porcentuales, con lo que se elevará a un 32\% en el primer caso y un $67 \%$ en el segundo. En Nicaragua la deuda permanecerá prácticamente igual en relación con el PIB; presentará solo 2 puntos porcentuales de alza y llegará al 38\%.

Este aumento extraordinario de la deuda pública responde a la ampliación del déficit fiscal. En efecto, en todos los países dicho balance muestra un incremento del déficit -en comparación con el aprobado previamente en el presupuesto-, que va de menos de 1 punto porcentual en Costa Rica $(-0,4 \%)$ y Nicaragua $(-0,9 \%)$ a menos de 4 puntos porcentuales o alrededor de esa cifra en Panamá $(-3,1 \%)$, Guatemala $(-3,3 \%)$ y Honduras $(-4,4 \%)$, y alcanza un nivel mucho mayor, del $10 \%$, en El Salvador. Tales aumentos del déficit fiscal, con la salvedad de Costa Rica, responden más al incremento de los gastos que a la reducción de los ingresos tributarios. La pérdida esperada de dichos ingresos a lo largo de 2020 es pequeña y se ubica entre el 0,2\% y el 1,1\% del PIB correspondiente (ICEFI, 2020b). Estas contracciones no son meramente resultantes de la caída de la actividad; reflejan la aplicación 
de incentivos fiscales con orientación contracíclica. El exceso de gastos (si puede llamarse asi) por encima de los inicialmente considerados presenta variaciones menos homogéneas en la subregión. Las estimaciones del ICEFI (2020b) ubican este "sobregasto" en medio punto porcentual del PIB en Costa Rica y Nicaragua, un nivel coherente con su expansión del déficit fiscal relativamente limitada. En los casos de Guatemala y Honduras el alza proyectada llega a 2,5 y 3,2 puntos porcentuales del PIB, respectivamente, en tanto que la mayor alza, de 6,5 puntos porcentuales, se produciría en El Salvador.

A continuación, se comentarán con más detalles algunas de las principales medidas fiscales aplicadas en este esfuerzo contracíclico en los países centroamericanos, en diferentes montos y momentos. Estas incluyen modificaciones temporales en la recolección tributaria mediante créditos fiscales, condonaciones, amnistías, exenciones, reducciones y aplazamiento de los pagos.

En primer lugar, queremos destacar que en esta contingencia se han otorgado apoyos fiscales orientados tanto a las personas y las familias como a empresas vulnerables o afectadas ${ }^{4}$. En cuanto a los apoyos a la población, en Costa Rica se realizaron transferencias a personas en condición de pobreza y se distribuyeron paquetes de alimentos y de artículos de bioseguridad a personas con discapacidad, en extrema pobreza, personas mayores o afectadas por el desempleo. En El Salvador se transfirieron 300 dólares por familia a 1,5 millones de hogares y se entregaron 1 millón de paquetes de alimentos. En Guatemala se otorgaron transferencias focalizadas de 130 dólares mensuales durante tres meses y se entregó un bono de apoyo al sector informal, a los pensionados y a trabajadores del sector de la salud. También se repartieron paquetes de alimentos, medicinas e insumos a personas vulnerables. En Honduras se realizaron transferencias a trabajadores del sector del transporte y se entregaron paquetes de alimentos y artículos de higiene personal. En Panamá se entregaron vales para la compra de alimentos, medicinas y gas en la zona urbana y paquetes con estos productos en la zona rural. En el caso de Nicaragua, la información oficial solo indica la entrega de paquetes de alimentos en diversas zonas del país. Como medida adicional, en algunos países se otorgaron subsidios al consumo de servicios básicos. También se tomaron medidas en el campo del trabajo; por ejemplo, en El Salvador se aprobó un aumento salarial de 150 dólares para los empleados públicos que realizan un trabajo directo en la lucha contra de la pandemia. En general, la expansión de los apoyos fiscales dirigidos a los hogares se concretó mediante transferencias en dinero o especie.

Además de las medidas para proteger el consumo de los hogares, se tomaron medidas dirigidas al sector empresarial con el fin de preservar el empleo y la capacidad productiva. Los instrumentos para ello han sido diversos. En Costa Rica se realizaron transferencias públicas temporales dirigidas a las microempresas y pequeñas y medianas empresas (mipymes) de los sectores pesquero, turístico y agrícola. También se estableció una suspensión temporal de los impuestos a los boletos aéreos que pagan las compañías de aviación, por cuatro meses, y de las cuotas anuales para el sector pesquero, por tres meses. En Guatemala se declaró una moratoria de tres meses para el pago del impuesto corporativo y se suspendió el pago de las contribuciones - también por tres meses - de los empleadores al Instituto de Recreación de los Trabajadores de la Empresa Privada de Guatemala (IRTRA), al Instituto Técnico de Capacitación y Productividad (INTECAP) y al Instituto Guatemalteco de Seguridad Social (IGSS). En Honduras se aplazó el pago de impuestos de las empresas consideradas contribuyentes pequeños y medianos. En Panamá se difirió el pago de todo tipo de impuestos por 120 días y en El Salvador se otorgaron subsidios a las empresas con 100 empleados o menos para cubrir el 50\% de los salarios por un máximo de hasta dos meses y, además, se difirió la contribución especial de las empresas turísticas. Aparte de las medidas fiscales, las empresas también recibieron apoyo financiero, aspecto que se discutirá con más detalle en los párrafos subsiguientes. El único país de la subregión que no concedió apoyos fiscales a las empresas es Nicaragua.

\footnotetext{
${ }^{4}$ Las medidas fiscales se recopilaron de las siguientes fuentes: Matarrita y Romero (2020), CEPAL (2020f), FMl (2020) y Ministerio de Hacienda de El Salvador (2020).
} 
Otro tipo de medidas fiscales sirvieron indistintamente a hogares y empresas. Por ejemplo, en Costa Rica se declaró una moratoria del pago del impuesto sobre la renta (ISR), el impuesto sobre el valor agregado (IVA) y aranceles, y se rebajó en un $75 \%$ la base mínima contributiva que se paga a la Caja Costarricense de Seguro Social (CCSS) en el caso de los patrones, trabajadores independientes y trabajadores del sector público. En El Salvador se aplazó temporalmente el pago del ISR para personas naturales y jurídicas. En Honduras se postergó el pago de todos los impuestos en el caso de los pequeños y medianos contribuyentes hasta junio de 2020 y del ISR hasta fines de agosto, además de establecerse una disminución del ISR del 8,5\% también para este tipo de contribuyentes, mientras que en Panamá se prorrogó el pago de tributos por 120 días.

Los Gobiernos de la subregión han requerido financiamiento adicional para dotar de fondos a los programas y modalidades especiales de respuesta a la doble emergencia. Entre las fuentes externas destacan el Fondo Monetario Internacional (FMI), el Banco Mundial, el Banco Interamericano de Desarrollo (BID) y el Banco Centroamericano de Integración Económica (BCIE). En la subregión en su conjunto, los desembolsos más grandes han sido efectuados por el FMI (2.145 millones de dólares) y el Banco Mundial (2.025 millones de dólares). El BCIE también ha realizado un elevado desembolso (1.453 millones de dólares). De acuerdo con CEPAL (2020a), los países que más han emitido deuda son Costa Rica (1.679 millones de dólares), Honduras (1.169 millones de dólares) y Panamá (1.065 millones de dólares).

Una limitación de la estrategia inicial ante la emergencia es que los planes de iniciativas fiscales se concentraron -como sería previsible- en las tareas que debían acometerse en el corto plazo y descuidaron, por así decirlo, el diseño de una estrategia posterior a la emergencia para garantizar la sostenibilidad de la pauta de endeudamiento, en coherencia con una nueva agenda de desarrollo sostenible e incluyente de largo plazo.

Los proyectos de los presupuestos de ingresos y gastos públicos para 2021 deberían incluir planes que promuevan una recuperación económica sostenida y sostenible en el mediano y largo plazo. Hasta septiembre de 2020, solamente Costa Rica, Guatemala, Honduras y Panamá han presentado su proyecto de presupuesto para $2021^{5}$. En los casos de Guatemala y Honduras, se incluyen criterios multianuales hasta 2023; sin embargo, los montos estipulados del esfuerzo fiscal para atender las consecuencias de la crisis en el mediano plazo pueden llegar a ser insuficientes ${ }^{6}$. Además, en ambos casos se planea recurrir a la consolidación fiscal para subsanar el impacto sobre las finanzas públicas. Costa Rica ha propuesto fuertes medidas de consolidación fiscal para 2021 y en septiembre se encontraba negociando un acuerdo con el FMI que implica reducciones importantes del gasto público y venta de activos en el mediano plazo. Es preciso alertar en cuanto a que la subregión corre el riesgo de un retorno muy prematuro a políticas de austeridad, que alargarían y profundizarían la recesión, precarizarían más el mercado laboral y, con ello, aumentarían los niveles de pobreza y desigualdad.

En el ámbito de la política monetaria y crediticia, los países de Centroamérica reaccionaron principalmente con disminuciones de la tasa de interés, reducciones del encaje bancario y medidas orientadas a facilitar el acceso al crédito $^{7}$. Los bajos niveles de inflación brindan espacio para la ejecución de políticas monetarias convencionales de carácter expansivo. Según datos de la Secretaría Ejecutiva del Consejo Monetario Centroamericano (SECMCA, 2020), entre febrero y septiembre de 2020 Costa Rica decidió reducir la tasa en 450 puntos básicos (del 5,25\% al 0,75\%), Guatemala en 100 puntos básicos (del 2,75\% al 1,75\%) y Honduras en 150 puntos básicos (del 5,25\% al 3,75\%).

5 Véase Ministerio de Hacienda de Costa Rica (2020), Secretaría de Finanzas de Honduras (2020a y 2020b), Ministerio de Finanzas Públicas de Guatemala (2020) y Ministerio de Economía y Finanzas de Panamá (2020).

6 En el caso de Guatemala, en el Proyecto de Presupuesto General de Ingresos y Egresos del Estado Ejercicio Fiscal 2021 se detalla que el costo de las medidas extraordinarias propuestas para mitigar los daños ocasionados por la crisis representa el 2,9\% del monto presupuestado para el año (véase Ministerio de Finanzas Públicas de Guatemala, 2020).

7 Las medidas monetarias y crediticias se recopilaron de las siguientes fuentes: Matarrita y Romero (2020), CEPAL (2020f), FMl (2020), Banco de Guatemala (2020) y Banco Central de Honduras (2020). 
Nicaragua, por su parte, fijó la tasa de política monetaria en un 6\% en abril y la había reducido en 175 puntos básicos en agosto de 2020.

Por otro lado, en algunos países se realizaron modificaciones al encaje bancario, con el objetivo de aumentar la liquidez o incentivar la compra de deuda pública por parte del sistema bancario interno. En Guatemala se aprobó la flexibilización del cómputo del encaje bancario por un plazo de 180 días; en Nicaragua se disminuyó el encaje legal en un 10,5\%, una medida que estará vigente durante 2020, y, según datos de la SECMCA (2020), en El Salvador se redujo el encaje bancario efectivo del 22,8\% en febrero al 16,6\% en julio de 2020. Entre otras medidas implementadas para inyectar liquidez a la economía, resalta la compra de bonos del gobierno en el mercado secundario por parte del Banco Central de Costa Rica hasta por un monto de 250.000 millones de colones. En relación con medidas vinculadas al tipo de cambio, solamente en Guatemala se intervino en el mercado de divisas para mantener la estabilidad cambiaria, por un monto de 400 millones de dólares, en marzo de 2020.

Dado que la inflación es persistentemente baja, las reducciones de la tasa de interés y las inyecciones de liquidez se producen en términos reales. Sin embargo, hay que tener en cuenta que la efectividad de los instrumentos convencionales de la política monetaria expansiva en períodos de crisis es muy baja tanto por las deterioradas expectativas de los inversionistas sobre sus rendimientos futuros como por la alta concentración y la escasa profundidad de los mercados financieros.

Como complemento a la política monetaria expansiva, en la mayoría de los países se han implementado medidas para facilitar el acceso al crédito y condiciones más atractivas para los hogares y las empresas afectadas. En Costa Rica se aprobó una línea especial de crédito para intermediarios financieros por 700.000 millones de colones y otra línea dedicada a las mipymes para capital semilla, compra de insumos intermedios o pago de planillas; en El Salvador se creó un fondo fiduciario de 600 millones de dólares para el apoyo de las mipymes y el sector informal; en Honduras también se instauró un fondo fiduciario para brindar crédito a las mipymes; en Guatemala se estableció una línea por 100 millones de quetzales para las mipymes, y en Panamá se creó un fondo especial para fortalecer el acceso al crédito, destinando 500 millones de dólares a la estabilidad del sistema financiero y otros 500 millones de dólares al establecimiento de líneas de crédito destinadas a los sectores más afectados por la pandemia. Entre las medidas crediticias, en varios países se aplicaron moratorias y se reestructuraron préstamos personales y empresariales, así como también se procedió al congelamiento y la regularización de las calificaciones crediticias. Por ejemplo, en Costa Rica se suspendió temporalmente la cobranza del interés en el caso de clientes afectados por la crisis que tienen créditos hipotecarios en el Instituto Nacional de Vivienda y Urbanismo (INVU) y en El Salvador se apoyó financieramente al sector agrícola por medio de una moratoria del pago de los préstamos bancarios.

Además de las medidas macroeconómicas, se aplicaron instrumentos complementarios para, por ejemplo, controlar los precios de productos clave o intervenir directamente con regulaciones nuevas en el mercado laboral. Con excepción de Nicaragua, en todos los países se impusieron controles de precios de productos de la canasta básica. En algunos también se controlaron los precios de los productos de higiene personal y bioseguridad. En relación con las intervenciones directas en el mercado laboral, resalta la creación en Costa Rica de un subsidio temporal para trabajadores afectados por la emergencia sanitaria y la aprobación en El Salvador de la Ley de Protección al Empleo Salvadoreño ${ }^{8}$, que comprende medidas temporales para salvaguardar la estabilidad de los empleados del sector privado.

La implementación de las políticas económicas para contrarrestar el impacto negativo de la crisis sobre las economías centroamericanas requiere de un esfuerzo fiscal considerable ${ }^{9}$. El rango de la magnitud del esfuerzo fiscal anunciado por los Gobiernos abarca del 0,8\% del PIB en Costa Rica al

\footnotetext{
8 Véase [en línea] https://www.diariooficial.gob.sv/diarios/do-2020/05-mayo/05-05-2020.pdf.

9 El monto final de la ejecución presupuestaria puede variar debido a las condiciones adversas del acceso al financiamiento externo. Además, la emisión de deuda pública está sujeta a la aprobación de los Congresos.
} 
$11,1 \%$ del PIB en El Salvador. En Honduras, este porcentaje asciende al 4,3\% del PIB, en Guatemala al 2,5\% y en Panamá al 3,7\% (CEPAL, 2020d). Para Nicaragua no hay datos, ya que no se presentó un programa oficial de estímulo fiscal.

El tamaño del esfuerzo realizado no está necesariamente relacionado con el espacio fiscal que se percibía a priori. Costa Rica y El Salvador presentan mayores dificultades en sus finanzas públicas, con niveles de deuda pública -como proporción del PIB - superiores al 70\% en 2019 (ICEFI, 2020a), y el monto de sus paquetes fiscales en relación con el PIB es contrastante. El éxito de las medidas contracíclicas no depende solo del monto final de la ejecución, sino también de su composición y de su aplicación oportuna. Sin duda es necesaria una planificación adecuada por medio de registros estadísticos confiables y pertinentes, y una ejecución transparente que incluya la rendición de cuentas del uso del dinero público.

La crisis obliga a una coordinación cercana entre las políticas fiscal y monetaria. La política fiscal debe ejercer el rol de liderazgo en el impulso a la actividad productiva y, en forma paralela, la política monetaria debe garantizar el financiamiento en el tiempo del aumento inevitable del déficit fiscal al menor costo posible (Panico, 2020). En Centroamérica, El Salvador y Panamá poseen economías dolarizadas, lo que imposibilita la coordinación entre las políticas. En cambio, aunque en magnitudes diversas, en Costa Rica, Guatemala y Honduras se han implementado políticas fiscales y monetarias expansivas de manera coordinada para abordar los efectos de la crisis.

Subrayamos que la política fiscal debe ejercer ese liderazgo, no solo en el corto plazo para afrontar las dificultades inmediatas, sino también en el mediano y el largo plazo, para contribuir a una recuperación económica robusta e incluyente. Para ello, es necesario considerar la necesidad de implementar reformas fiscales progresivas y redistributivas, y de reducir al máximo la elusión y la evasión de impuestos, con el objetivo de atender de manera integral el deterioro de las finanzas públicas. Igualmente importante es el diseño y la ejecución de medidas para superar las restricciones externas al crecimiento, tales como la regulación de los flujos de capitales y una política de transformación productiva que permita aumentar el valor agregado contenido en las exportaciones.

No obstante el esfuerzo en materia de políticas contracíclicas, será imposible compensar totalmente el inmenso golpe que ha significado esta doble crisis. Así, las estimaciones sobre el comportamiento de la actividad económica y la incidencia de la pobreza y la desigualdad para 2020 no son alentadoras. La evolución del índice mensual de actividad económica (IMAE) de los países centroamericanos ya muestra una importante tendencia a la baja. De acuerdo con información de la SECMCA (2020), la caída interanual registrada hasta mediados de 2020 (según el último dato disponible para cada país a septiembre) alcanza en Nicaragua un 9,5\% en abril; en Panamá un 40,9\% en mayo; en El Salvador un 12,2\% y en Honduras un $13,4 \%$ en junio; en Costa Rica un $8,1 \%$ y en Guatemala un 4,8\% en julio. Se espera que con el relajamiento de las medidas de confinamiento los países recuperen paulatinamente el ritmo de su actividad económica; sin embargo, no se descarta un segundo brote de contagios que, de ocurrir, tendría un impacto devastador.

Según las proyecciones de la CEPAL (2020a), se produciría una caída pronunciada del PIB real en todos los países de la subregión (véase el cuadro 4). Se estima una contracción promedio del 5,9\% para Centroamérica, mucho menor que la esperada para el conjunto de América Latina y el Caribe, del 9,1\%. Las caídas más agudas en la subregión se registrarían en El Salvador (-8,6\%) y Nicaragua (-8,3\%); esta última es muy preocupante, dado que profundizaría la crisis iniciada dos años atrás. Un indicador alternativo del efecto de la pandemia en la pauta de expansión de las economías, quizá más revelador, es la diferencia entre la tasa de crecimiento de 2019 y la proyectada para 2020. Desde esta óptica, se advierte que la pandemia provocará, al parecer, una desaceleración muy similar en Centroamérica y en América Latina y el Caribe en promedio. Efectivamente, mientras que la tasa de expansión del PIB de Centroamérica caerá 8,4 puntos porcentuales, la de América Latina y el Caribe disminuirá 9,1 puntos porcentuales. Desaceleraciones profundas se observarían en todos los 
países: El Salvador (-11,0 puntos porcentuales), Panamá $(-9,5)$, Honduras $(-8,8)$, Guatemala $(-7,9)$ y Costa Rica $(-7,6)$. Nicaragua, en este sentido, es el país que mostraría un menor registro, pero partiendo ya de una recesión considerable en 2019. Es indudable que el impacto de la pandemia en la actividad económica sería mucho más agudo, de no ser por las políticas contracíclicas aplicadas.

Cuadro 4

Centroamérica: tasa de crecimiento del PIB real, 2019 y 2020

(En porcentajes)

\begin{tabular}{lccc}
\hline País & 2019 & $2^{2020^{\mathrm{a}}}$ & $\begin{array}{c}\text { Diferencia 2019-2020 } \\
\text { (puntos porcentuales) }^{\mathrm{a}}\end{array}$ \\
\hline Centroamérica & 2,5 & $-5,9$ & 8,4 \\
\hline Costa Rica & 2,1 & $-5,5$ & 7,6 \\
\hline El Salvador & 2,4 & $-8,6$ & 11,0 \\
\hline Guatemala & 3,8 & $-4,1$ & 7,9 \\
\hline Honduras & 2,7 & $-6,1$ & 8,8 \\
\hline Picaragua & $-3,9$ & $-8,3$ & 4,4 \\
\hline América Latina y el Caribe & 3,0 & $-6,5$ & 9,5 \\
\hline
\end{tabular}

Fuente: Elaboración propia, sobre la base de Comisión Económica para América Latina y el Caribe (CEPAL), CEPALSTAT, 2020 [base de datos en línea] http://interwp.cepal.org/cepalstat/Portada.html?idioma=spanish; Estudio económico de Centroamérica y la República Dominicana en 2019 y perspectivas para 2020 (LC/MEX/TS.2020/29), Ciudad de México, 2020, y "Enfrentar los efectos cada vez mayores del COVID-19 para una reactivación con igualdad: nuevas proyecciones", Informe Especial COVID-19, № 5, Santiago, 15 de julio, 2020.

a Proyecciones de la CEPAL.

En cuanto a la evolución del PIB real per cápita, las proyecciones de la CEPAL (2020a) señalan una caída del 7,1\% en 2020 en la subregión en su conjunto, con las disminuciones más importantes en El Salvador $(-9,4 \%)$ y Nicaragua $(-9,2 \%)$. La contracción es tan grande que pasarán años antes de que Centroamérica logre recuperar los niveles anteriores a la pandemia. The Economist Intelligence Unit proyecta que El Salvador y Guatemala podrían no recuperar el nivel del PIB de 2019 hasta 2023 (The Economist, 2020a y 2020b). En el caso de Nicaragua, las estimaciones indican que aún no habrá recuperado el PIB de 2017 -anterior a la crisis sociopolítica de 2018 - en 2024 (The Economist, 2020c).

En cuanto a los efectos esperados sobre la incidencia de la pobreza, el cuadro 5 presenta proyecciones para 2020. Se prevén incrementos de los niveles de pobreza y pobreza extrema en todos los países de la subregión. En Guatemala, El Salvador, Honduras y Nicaragua, los aumentos serían incluso mayores que los estimados para el conjunto de América Latina.

El Salvador y Nicaragua también son los países en los que se espera un aumento mayor de la incidencia de la pobreza y la pobreza extrema. El retroceso será grave y significará perder los logros obtenidos en los últimos años en la lucha contra la pobreza. Según las proyecciones, en 2020 la incidencia de la pobreza en Costa Rica será similar a la de 2007, en El Salvador a la de 2015, en Guatemala a la de 2014, en Honduras a la de 2013, en Nicaragua a la de 2009 y en Panamá a la de 2015 (CEPAL, 2020d). Las proyecciones también indican que la desigualdad aumentará en todos los países. En El Salvador, se proyecta un incremento del coeficiente de Gini en un porcentaje ubicado entre el 5,0\% y el 5,9\%; en Costa Rica y Panamá, entre el 3,0\% y el 3,9\%; en Honduras y Nicaragua, entre el 2,0\% y el 2,9\%, y en Guatemala entre el 1,0\% y el 1,9\% (CEPAL, 2020d). En general, Nicaragua y El Salvador son los países que se verán afectados en mayor medida por la crisis en cuanto a reducciones del crecimiento económico y aumentos de la incidencia de la pobreza. 


\section{Cuadro 5}

Centroamérica: población en situación de pobreza y de pobreza extrema, 2019 y 2020 (En porcentajes)

\begin{tabular}{|c|c|c|c|c|}
\hline Indicador & País & 2019 & $2020^{\mathrm{a}}$ & $\begin{array}{l}\text { Diferencia 2020-2019 } \\
\text { (puntos porcentuales) }\end{array}$ \\
\hline \multirow{7}{*}{ Pobreza } & América Latina & 30,2 & 37,3 & 7,1 \\
\hline & Costa Rica & 16,5 & 20,5 & 4,0 \\
\hline & El Salvador & 33,7 & 40,2 & 6,5 \\
\hline & Guatemala & 48,6 & 51,6 & 3,0 \\
\hline & Honduras & 54,8 & 59,0 & 4,2 \\
\hline & Nicaragua & 47,1 & 52,7 & 5,6 \\
\hline & Panamá & 14,6 & 17,5 & 2,9 \\
\hline \multirow{7}{*}{ Pobreza extrema } & América Latina & 11,0 & 15,5 & 4,5 \\
\hline & Costa Rica & 3,4 & 5,1 & 1,7 \\
\hline & El Salvador & 7,4 & 11,9 & 4,5 \\
\hline & Guatemala & 19,8 & 22,7 & 2,9 \\
\hline & Honduras & 18,7 & 22,2 & 3,5 \\
\hline & Nicaragua & 18,0 & 22,8 & 4,8 \\
\hline & Panamá & 6,5 & 8,5 & 2,0 \\
\hline
\end{tabular}

Fuente: Elaboración propia, sobre la base de Comisión Económica para América Latina y el Caribe (CEPAL), "Enfrentar los efectos cada vez mayores del COVID-19 para una reactivación con igualdad: nuevas proyecciones", Informe Especial COVID-19, № 5, Santiago, 15 de julio de 2020

a Proyecciones de la CEPAL.

Las proyecciones sobre el crecimiento económico, la pobreza y la desigualdad llevan a reflexionar acerca de la relevancia de la política contracíclica. Si bien las medidas adoptadas fueron relevantes, en general, se necesita un esfuerzo mucho mayor que el realizado hasta el momento. En Costa Rica el esfuerzo - como proporción del PIB - ha sido insuficiente, y en Nicaragua nulo. Dado el impacto de la crisis y las perspectivas para el futuro, preocupa que los países retornen a políticas de austeridad demasiado pronto. Lo ideal es que la política fiscal contracíclica se institucionalice mediante la creación de estabilizadores automáticos. Además, las medidas deben enmarcarse en una visión de futuro y orientarse a preservar el empleo, proteger a la población vulnerable y promover una transformación productiva que asegure la sostenibilidad ambiental y la inclusión social. Sobre este punto, ahondaremos más en el siguiente apartado.

\section{Reflexiones finales}

Los Gobiernos de la subregión tuvieron una pronta reacción para enfrentar el golpe brutal que ha significado la pandemia. Inicialmente, además de ordenarse confinamientos y distanciamiento físico, se dieron apoyos para atender la salud y el bienestar material de las familias y empresas. Una vez relajadas las medidas de confinamiento, los Ministerios de Hacienda en coordinación con los bancos centrales y entidades de los gabinetes económicos y sociales hicieron suya la frase de Keynes en el sentido de que el momento de la austeridad es el del auge económico, no el de la recesión ${ }^{10}$. Así, con la excepción de Nicaragua, pusieron en marcha - en diferentes grados y montos - programas de expansión del gasto público y de relajamiento de las condiciones monetarias y financieras. La gama de acciones aplicadas a la fecha es muy amplia; sin embargo, será insuficiente para lograr una recuperación robusta. Las proyecciones de las trayectorias del PIB para 2020 y 2021 se han revisado repetidamente a la baja, lo que hace prever una recesión profunda y una lenta recuperación. Las revisiones seguirán estando sujetas a la incierta evolución de la pandemia y a la continuación o no de las medidas contracíclicas.

\footnotetext{
10 "La austeridad fiscal y el recorte de gastos de las empresas no harán más que empeorar las condiciones preexistentes en el mundo" (Kozul-Wright, citado en UNCTAD, 2020b).
} 
Si bien la incertidumbre afecta las proyecciones de ingresos y gastos públicos, no hay duda de que el balance fiscal primario y global se deteriorará y que el peso de la deuda subirá, afectado, además, por la contracción del PIB, el rezago de los ingresos tributarios frente a los gastos de emergencia y las depreciaciones del tipo de cambio. Es indiscutible que en 2021 el espacio fiscal en cada país de la subregión será mucho más estrecho que el que tenían o el que crearon en 2020. Este menor margen de maniobra fiscal coincidirá con necesidades de la población mucho más agudas. Baste tener presente que a fines de 2020, el PIB per cápita promedio en la subregión habrá retrocedido en términos reales al nivel que registró hace diez o más años, millones de personas habrán perdido su trabajo formal y muchas más estarán afectadas por la caída de sus ingresos y se encontrarán en una situación por debajo de la línea de pobreza. Además, no hay indicio alguno de una recuperación económica en 2021, salvo quizá en China y algunos otros países asiáticos donde el repunte adquiera la fuerza suficiente para compensar la contracción actual.

En este escenario, se presentan grandes desafíos en la subregión en cuanto a su política macroeconómica y social de corto plazo, así como su pauta de desarrollo de largo plazo. El primer desafío que enfrenta es el de no retirar anticipadamente los estímulos fiscales, monetarios y financieros contracíclicos dirigidos tanto a la población vulnerable como a la inversión, la actividad productiva y la preservación de fuentes de trabajo del sector empresarial ${ }^{11}$. Sobran experiencias negativas en que el giro prematuro a la austeridad, antes de haber consolidado el repunte económico, más bien ha prolongado la recesión y profundizado la pobreza y la desigualdad. Una premisa central de la políica macroeconómica debe ser que una consolidación fiscal sostenible solo puede conseguirse en el marco de una robusta recuperación de la actividad económica. Buscar dicha consolidación, como en el pasado, priorizando la austeridad, con contención o recortes del gasto público, es contraproducente y muy costoso social y económicamente. En la medida en que agudiza y prolonga la recesión, tiende a aumentar - en vez de reducir - el cociente entre deuda y PIB ${ }^{12}$. Los Gobiernos de la subregión deben tomar en cuenta esta consideración, teniendo presente que, sin haber superado plenamente el primer brote de contagios de COVID-19, un segundo rebrote ya se percibe en varios países.

Enfrentar este primer reto implica un compromiso en materia fiscal para al menos mantener los estímulos de gasto público en esta fase de recesión, y hacerlos más eficaces y eficientes. Implica, a la vez, emprender acciones, o al menos sentar compromisos, para robustecer los ingresos tributarios y no tributarios y contemplar un programa de respaldo con financiamiento internacional. En cuanto a las iniciativas de gasto contracíclico, es imperativo que sus multiplicadores de ingreso y empleo sean elevados, sin que presionen excesivamente la balanza de pagos. En cuanto a los ingresos, la subregión adolece de una carga tributaria baja, con escaso impacto en términos de una redistribución más progresiva del ingreso. El mayor endeudamiento deberá considerarse en un horizonte temporal de al menos mediano plazo, a fin de asegurar que su trayectoria de sostenibilidad no se salga de control. Enfrentar este reto condiciona el grado en que los países de la subregión podrán sostener el esfuerzo contracíclico, e incide en las posibilidades de crear las condiciones para un repunte económico robusto. La debilidad estructural de los ingresos fiscales ha redundado en que la inversión pública haya sido el componente de ajuste ante las presiones por la consolidación fiscal, en detrimento de la infraestructura, del dinamismo de la inversión privada y, en síntesis, de la tasa potencial de expansión de las economías centroamericanas. En este esfuerzo, se necesita de la coordinación con las autoridades monetarias. Centroamérica deberá sopesar las posibilidades de que la banca central tenga un rol más activo en el financiamiento del desarrollo, más explícitamente de la inversión pública, y quizá en algunos proyectos

\footnotetext{
${ }^{11}$ De acuerdo con la Conferencia de las Naciones Unidas sobre Comercio y Desarrollo (UNCTAD, 2020a), retomar en 2021 la política de austeridad reducirá el crecimiento de la actividad productiva, aumentará el desempleo y la brecha laboral, y disminuirá la participación de los ingresos laborales.

12 Véase una crítica fundamentada - especialmente pertinente para Centroamérica y las economías en desarrollo- de la visión de austeridad expansiva propuesta, entre otros, por Alesina, Favero y Giavazzi (2020) en Skidelsky y Fraccaroli (2017) y Skidelsky (2019).
} 
en asociación con la actividad privada. Como señalamos en Barbosa y otros (2020): "tal financiamiento de la deuda pública puede conllevar riesgos para la estabilización macro de mediano plazo. Pero este es menor que la amenaza a la subsistencia de la población y que el riesgo a la paz social".

El segundo, no desligado del anterior, es un desafío mayúsculo de economía política. Se necesita crear un consenso entre los actores políticos, sociales y económicos relevantes - de la nación y luego de la subregión - para rediseñar la agenda de desarrollo. Tal como la emergencia inédita exigió respuestas inéditas de política económica y social, para dejar atrás la emergencia de la pandemia y la crisis económica, y situar a Centroamérica en una senda de crecimiento elevado y persistente de la actividad productiva y del empleo se requieren pactos políticos y estrategias económicas inéditas. No se puede volver al statu quo anterior a la crisis. Es impensable mantener la misma pauta de acumulación, producción y distribución. Es negligente e irresponsable con la problemática ambiental, la desigualdad y la exclusión. Esta pauta ha probado su incapacidad para remover la restricción de la balanza de pagos sobre el crecimiento económico de la subregión; al contrario, la ha acentuado. La reactivación de la subregión tendrá que ser sostenible ambientalmente e incluyente socialmente. De lo contrario, estará condenada a sufrir intermitentemente la explosión de crisis sanitarias, financieras y fiscales, sin descartar la posibilidad de que derive en inestabilidad política y social.

Como afirma Mukhisa Kituyi, Secretario General de la Conferencia de las Naciones Unidas sobre Comercio y Desarrollo (UNCTAD): "la construcción de un mundo mejor requiere acciones acertadas ahora. Las vidas de las generaciones futuras, y de hecho del propio planeta, dependerán de las decisiones que todos tomemos en los próximos meses" (Kituyi, citado en UNCTAD, 2020b). Esta construcción del futuro debe comenzar ya en plena lucha contra la pandemia. El corto plazo y el largo plazo son distintos, pero comienzan el mismo día, hoy. Una forma de que las políticas contracíclicas contribuyan es que los apoyos destinados a las empresas se sujeten a condiciones en materia de desempeño ambiental y social (véase UNCTAD, 2020a). De manera análoga, los proyectos de inversión que emprenda o promueva el sector público en su rol contracíclico deben orientarse a cimentar la transformación productiva de largo plazo que reclama la subregión. Un elemento central debe ser la vinculación de la aplicación de dichas políticas con la concertación de una reforma fiscal de fondo - que puede concretarse ya en la fase de recuperación - para robustecer el espacio fiscal y tener un Estado capaz de cumplir con sus responsabilidades esenciales en términos de protección social y desarrollo económico. En una visión más amplia, la pandemia y sus impactos potenciados por las desigualdades y deficiencias del modo de acumulación e inserción de Centroamérica en la economía globalizada deben aprovecharse para lanzar un pacto nacional por un desarrollo sostenible e igualitario, basado en una cultura de derechos.

Más allá del esfuerzo nacional, en el caso centroamericano la nueva agenda de desarrollo pasa ineludiblemente por revitalizar y potenciar las iniciativas de cooperación e integración regional. ¿De qué otra manera podrían lograrlo una serie de economías pequeñas, sumamente abiertas al comercio y a los mercados de capitales mundiales, con mercados internos muy reducidos y cuyos sectores productivos y sistemas de innovación distan de estar en la punta de las cadenas globales de valor? La integración centroamericana, a pesar de obstáculos y algunos retrocesos, es desde hace tiempo muy relevante. Baste señalar que casi un tercio de su comercio es intrarregional. Imprimirle mayor dinamismo es vital para avanzar hacia un desarrollo sostenible e incluyente. Desde ya se podrían dar pasos en esa dirección, en la aplicación de las políticas contracíclicas, centrando las inversiones y estímulos en acciones o áreas estratégicas, para reconvertir la matriz productiva en el sentido de la sostenibilidad ambiental con igualdad. La transición hacia la llamada economía verde abre vastas áreas de cooperación e inversión a la actividad privada y pública coordinada regionalmente para desencadenar y sostener un nuevo ciclo de transformación productiva y expansión económica. La lista es casi infinita. Por señalar algunas áreas, se podría llegar a acuerdos tributarios, de atracción selectiva de inversión extranjera directa, circulación de capitales de corto plazo, migración y producción estratégica de determinados productos, entre otros y, con visión de más largo alcance, acuerdos para modernizar la infraestructura del sistema de salud, energías limpias, transporte público, ciencia, tecnología e innovación y coordinación fiscal, entre otros. 
Pero, aun logrando una pronta revitalización del proceso de integración, la subregión no puede sola, sin un cambio en el concierto económico y financiero internacional. Los ingresos y gastos del sector público son muy limitados y están lejos de cubrir las necesidades de protección social e impulso al desarrollo. Solo con un respaldo internacional considerable Centroamérica podrá salir de la trampa de estancamiento, desigualdad y deterioro ambiental en que se encuentra sumida. El respaldo debe cubrir dos grandes aspectos. Se requiere, por una parte, una recuperación de la economía y el comercio mundial y, por otra, recursos de los organismos financieros internacionales. Para lo primero, es indispensable que las políticas macroeconómicas del mundo desarrollado mantengan su decidido impulso fiscal y monetario hasta que el consumo, y sobre todo la inversión privada, cobre dinamismo. Para lo segundo, es necesario que los organismos correspondientes tengan el convencimiento político y los fondos para dar respaldo financiero a Centroamérica. Como señaló recientemente la UNCTAD (2020a y 2020b), el mundo en desarrollo enfrenta un déficit de entre 2 y 3 billones de dólares en necesidades de financiamiento para los próximos 18 meses a causa de la pandemia.

En cuanto a recomendaciones más concretas sobre la arquitectura financiera internacional necesaria para salir de la pandemia con pasos firmes mediante una nueva agenda de desarrollo sostenible, sustentable e incluyente para Centroamérica, queremos subrayar la pertinencia de cuatro propuestas recientes de la UNCTAD: i) ampliar significativamente el uso de los derechos especiales de giro para apoyar las estrategias nacionales de los países en desarrollo; ii) lanzar a la brevedad una suerte de Plan Marshall para la recuperación de la salud mundial, financiado de manera tripartita con recursos de la cooperación para el desarrollo y de organismos multilaterales, junto con un renovado esfuerzo en el marco del Proyecto sobre la Erosión de la Base Imponible y el Traslado de Beneficios (BEPS) para disminuir la elusión y la evasión tributaria; iii) crear una agencia multinacional de calificación crediticia bajo control público, que vendría a sustituir a las muy poderosas agencias existentes en la actualidad, con el fin de disponer de calificaciones mucho más objetivas, que tomen en cuenta la perspectiva tanto de las corporaciones financieras como de los países en desarrollo, y iv) crear una autoridad mundial de la deuda para establecer un marco jurídico e institucional internacional amplio y transparente que regiría las suspensiones temporales automáticas de los reembolsos de la deuda soberana en tiempos de crisis y gestionaría las reestructuraciones de manera justa, eficiente y transparente ${ }^{13}$.

Para concluir, subrayaremos dos lecciones fundamentales de la presente pandemia. La primera es la necesidad de recuperar la legitimidad del Estado como actor esencial e insustituible en la definición de la agenda de desarrollo, la identificación de los obstáculos o restricciones clave y la movilización de recursos para salvarlos. La segunda es la de revalorizar la noción de lo público frente a lo privado. Estos meses, en que se han vivido situaciones muy dramáticas en muchas ciudades y pueblos de la subregión y del mundo, nos han recordado que la salud de todos depende de la de los más vulnerables. La pandemia hizo patente en la subregión la ausencia de un régimen que pueda medio llamarse de bienestar ${ }^{14}$. Dejó al desnudo lacerantes desigualdades en algo tan básico como el acceso a agua potable y alimentación adecuada, por no mencionar la calidad y el acceso a educación, salud y empleo. Estas desigualdades son heridas sociales cuya raíz viene de inequidades de mucho tiempo atrás en la distribución de los beneficios del crecimiento. Estas heridas se reabren cada día, ante el cómplice silencio e indiferencia de los que más tienen, y con la pandemia se han expuesto de manera abrupta y dramática. Frente a ello, es imperativo construir este pacto nacional -y regionalcon las características antes señaladas. Sin este pacto, el porvenir de Centroamérica se verá truncado y cancelado para los vastos contingentes de su población pobre, marginada y vulnerable, y en ese caso también se cancelará para todos como sociedad civilizada.

\footnotetext{
13 Véase una descripción detallada de estas iniciativas en UNCTAD (2020a).

${ }^{14}$ Véase una aproximación analítica a las características que debe tener un régimen de bienestar, con referencia al caso centroamericano y de México, en CEPAL (2020a y 2020h).
} 


\section{Bibliografía}

Alesina, A., C. Favero y F. Giavazzi (2020), Austeridad: cuándo funciona y cuándo no, Barcelona, Ediciones Deusto. Banco Central de Honduras (2020), "El Banco Central de Honduras revisa el programa monetario 2020-2021 y aprueba una nueva reducción en su tasa de política", Boletín de Prensa, № 42/2020, Tegucigalpa, 31 de julio.

Banco Central de la República Dominicana (2020), "Entorno internacional” [en línea] https://www.bancentral. gov.do/a/d/2585-entorno-internacional.

Banco de Guatemala (2020), "La Junta Monetaria por unanimidad reduce en 25 puntos básicos la tasa de interés líder de política monetaria", Boletín de Prensa, Ciudad de Guatemala, 25 de junio.

Banco Mundial (2020), Informe Semestral de la Región América Latina y el Caribe: la economía en los tiempos del COVID-19, Washington, D.C., abril.

Barbosa, N. y otros (2020), "América Latina frente al Gran Apagón", El País, 6 de mayo [en línea] https:// elpais.com/economia/2020-05-06/america-latina-frente-al-gran-apagon.html.

Castellani, F. y otros (2020), El impacto del COVID-19 en las economías de la región, Banco Interamericano de Desarrollo (BID) [en línea] https://publications.iadb.org/es/el-impacto-del-covid-19-en-las-economiasde-la-region-centroamerica.

CEPAL (Comisión Económica para América Latina y el Caribe) (2020a), Estudio económico de Centroamérica y la República Dominicana en 2019 y perspectivas para 2020 (LC/MEX/TS.2020/29), Ciudad de México. (2020b), Panorama Fiscal de América Latina y el Caribe, 2020 (LC/PUB.2020/6-P), Santiago.

(2020c), "Los efectos del COVID-19 en el comercio internacional y la logística", Informe Especial COVID-19, $N^{\circ}$ 6, Santiago, 6 de agosto.

(2020d), "Enfrentar los efectos cada vez mayores del COVID-19 para una reactivación con igualdad: nuevas proyecciones", Informe Especial COVID-19, N 5, Santiago, 15 de julio.

(2020e), "Sectores y empresas frente al COVID-19: emergencia y reactivación", Informe Especial COVID-19, № 4, Santiago, 2 de julio.

(2020f), "El desafío social en tiempos del COVID-19", Informe Especial COVID-19, № 3, Santiago, 12 de mayo.

(2020g), "Observatorio COVID-19 en América Latina y el Caribe: impacto económico y social" [en línea] https://www.cepal.org/es/temas/covid-19.

(2020h), Aprender de la historia, atender la emergencia, repensar el futuro. México, Centroamérica y el Caribe frente a la pandemia: diagnóstico y perspectivas (LC/MEXTS.2020/17/Rev.2), Ciudad de México. (2020i), CEPALSTAT [base de datos en línea] http://interwp.cepal.org/cepalstat/Portada.html?idioma=spanish.

FMI (Fondo Monetario Internacional) (2020), "Policy Responses to COVID-19", [en línea] https://www.imf.org/ en/Topics/imf-and-covid19/Policy-Responses-to-COVID-19 [fecha de consulta: 18 de septiembre de 2020].

ICEFI (Instituto Centroamericano de Estudios Fiscales) (2020a), Perfiles Macrofiscales de Centroamérica, $N^{\circ} 13$, Guatemala, abril.

(2020b), "Estimación de la situación fiscal de Centroamérica al final de 2020", Notas de Coyuntura, $N^{\circ}$ 05-2020, Guatemala, julio.

Matarrita, A. y R. Romero (2020), "Políticas económicas implementadas en la región CAPARD para contrarrestar el impacto del COVID-19", Notas Económicas Regionales, № 114, San José, Secretaría Ejecutiva del Consejo Monetario Centroamericano (SECMCA), junio.

Ministerio de Economía y Finanzas de Panamá (2020), "MEF presenta proyecto de Presupuesto General del Estado 2021 por B/. 24.088.9 millones a la Asamblea Nacional”, 30 de julio [en línea] https://www. mef.gob.pa/2020/07/mef-presenta-proyecto-de-presupuesto-general-del-estado-2021-por-b-24-0889-millones-a-la-asamblea-nacional/.

Ministerio de Finanzas Públicas de Guatemala (2020), "Proyecto de Presupuesto" [en línea] https://www. minfin.gob.gt/index.php/proyecto-de-presupuesto.

Ministerio de Hacienda de Costa Rica (2020), "Presupuesto Nacional de la República" [en línea] https://www. hacienda.go.cr/contenido/12487-presupuesto-nacional-de-la-republica.

Ministerio de Hacienda de El Salvador (2020), Estimación de los costos económicos, sociales, fiscales y medidas adoptadas en el marco de la pandemia COVID-19, San Salvador, abril.

Mooney, H. y M. Zegarra (2020), "COVID-19: shock sin precedentes sobre el turismo en América Latina y el Caribe”, Resumen de Políticas, № 339, Washington, D.C., Banco Interamericano de Desarrollo (BID), julio. 
Panico, C. (2020), "La coordinación de las políticas económicas en los tiempos del coronavirus", Economía UNAM, N 51, Ciudad de México, Universidad Nacional Autónoma de México (UNAM).

SECMCA (Secretaría Ejecutiva del Consejo Monetario Centroamericano) (2020), SECMCADATOS [base de datos en línea] http://www.secmca.org/secmcadatos/.

Secretaría de Finanzas de Honduras (2020a), "Lineamientos de política presupuestaria 2021-2023" [en línea] https://www.sefin.gob.hn/formulacion-y-aprobacion/.

(2020b), "Normas de ejecución presupuestaria (disposiciones generales)" [en línea] https://www.sefin. gob.hn/formulacion-y-aprobacion/.

SIECA (Secretaría de Integración Económica Centroamericana) (2020), Sistema de Estadísticas de Comercio de Centroamérica [base de datos en línea] http://www.sec.sieca.int/.

Skidelsky, R. (2019), “¿La reivindicación de la austeridad?”, Project Syndicate, 22 de mayo [en línea] https:// www.project-syndicate.org/commentary/budget-deficits-austerity-growth-alesina-keynes-by-robertskidelsky-2019-05/spanish.

Skidelsky, R. y N. Fraccaroli (eds.) (2017), Austerity vs Stimulus: the Political Future of Economic Recovery, Cham, Springer International Publishing.

The Economist (2020a), "El Salvador" [en línea] https://country.eiu.com/el-salvador. (2020b), "Guatemala" [en línea] https://country.eiu.com/guatemala. (2020c), "Nicaragua" [en línea] https://country.eiu.com/nicaragua.

UNCTAD (Conferencia de las Naciones Unidas sobre Comercio y Desarrollo) (2020a), Trade and Development Report 2020 (UNCTAD/TDR/2020), Ginebra.

(2020b), "COVID-19: la UNCTAD advierte de una 'década perdida' si las políticas económicas se centran en la austeridad fiscal”, 22 de septiembre [en línea] https://unctad.org/es/press-material/covid-19-launctad-advierte-de-una-decada-perdida-si-las-politicas-economicas-se. 